\title{
Noise-seeded spatiotemporal modulation instability in normal dispersion
}

\author{
D. Salerno, ${ }^{1,2}$ O. Jedrkiewicz, ${ }^{1}$ J. Trull, ${ }^{3}$ G. Valiulis, ${ }^{4}$ A. Picozzi, ${ }^{5}$ and P. Di Trapani ${ }^{1}$ \\ ${ }^{1}$ INFM e Dipartimento di Fisica e Matematica, Universitá dell'Insubria, Via Valleggio 11, 22100 Como, Italy \\ ${ }^{2}$ Facoltá of Scienze, Universitá di Milano, Via Celoria 16, 20132 Milano, Italy \\ ${ }^{3}$ Departament de Fisica i Enginyeria Nuclear C/ Colom 1, Universitat Politecnica de Catalunya, O8222 Terrassa (Barcelona) Spain \\ ${ }^{4}$ Department of Quantum Electronics, Vilnius University, Sauletekio 9-2040 Vilnius, Lithuania \\ ${ }^{5}$ CNRS, Laboratoire de Physique de la Matire Condense, Université de Nice, France
}

(Received 21 May 2004; published 22 December 2004)

\begin{abstract}
In optical second-harmonic generation with normal dispersion, the virtually infinite bandwidth of the unbounded, hyperbolic, modulational instability leads to quenching of spatial multisoliton formation and to the occurrence of a catastrophic spatiotemporal breakup when an extended beam is left to interact with an extremely weak external noise with a coherence time much shorter than that of the pump.
\end{abstract}

DOI: 10.1103/PhysRevE.70.065603

PACS number(s): 42.65.Tg, 42.65.Sf

The noise-seeded instability of extended wave packets (WP) in conservative evolutional nonlinear systems is a general and relevant phenomenon in wave physics, whose main manifestations are the appearance of regular modulations, wave breakup and, eventually, localized or soliton-like substructures. The theory used for the description and interpretation of the resulting rich phenomenology is that of the modulational instability (MI) of plane and monochromatic waves, extensively introduced in the context of gravity waves in deep waters [1] and applied for several different systems including plasmas [2], electric circuits [3], BoseEinstein condensates (BEC) [4] and, of course, optics [5]. The usually addressed MI signature is the preferential noise amplification at a given, intensity-dependent (spatial or temporal) frequency, which causes regular modulation in the direct space and sidebands in the spectral domain. This feature suitably describes the MI of both one-dimensional (1D) systems and of multidimensional "elliptical" ones, i.e., those supporting equisign linear phase modulation in all the available dimensions. However, it is generally not adequate for "hyperbolic" systems, where opposite signs occur for different dimensions. The elliptical is the most frequently encountered regime in the case of matter waves in isotropic media. The hyperbolic, in contrast, is the typical case of optical WPs in normally dispersive bulk media, diffraction, and chromatic dispersion leading in this case to linear phase modulations with opposite signs. Recently, dispersion-management techniques based on the use of periodic potentials [6] have made the hyperbolic regime of great interest also for the BEC waves.

As clearly pointed out in a theoretical analysis of hyperbolic MI performed for Kerr nonlinearity in optics [7,8], and also evident from the analysis of the $X^{(2)}$-driven MI [9], the the key feature that distinguishes the hyperbolic (normal) from the elliptic (anomalous) instability regime is that the MI gain profile in the $k$ - $\omega$ space is unbounded in the first (and only in the first) case. Indeed, in the frame of the usually adopted parabolic approximation for the material dispersion, we should say that any fluctuation with arbitrarily large spatial and temporal frequency shift with respect to the carrier mode has to be amplified, provided that both shifts lie on the suitable hyperbolic surface in the $k-\omega$ domain.
The unbounded feature of the hyperbolic MI raises two relevant questions: (i) the first concerns the interpretation of the number of studies regarding spatial MI in multidimensional systems. Indeed, following an experimental MI demonstration in (anomalous dispersive) 1D-temporal optical fibers [10], a number of experiments have been performed in multidimensional (i.e., in planar wave guides or in bulk samples) normal-dispersion materials, addressing the 1D spatial breakup of extended beams driven by quadratic $[11,12]$ as well as cubic [13] ultrafast nonlinear response. Surprisingly enough, the results were interpreted in terms of the direct spatial analogous of the temporal MI of above, as if the temporal degree of freedom and so the hyperbolic nature of the instability, had not taken any part in the process. (ii) The second question concerns the scenario that one should expect when the system interacts with a very broadband (i.e., virtually $\delta$-correlated) noise. In this case, in fact, the unbounded feature of the MI gain should lead one to forecast a catastrophic break in the spatio temporal (ST) domain (e.g., down to the numerical grid in calculations, in the quoted approximation), no matter how weak the input noise is. We note that the possible occurrence of such catastrophic dynamics has never been considered in the literature. In fact, hyperbolic MI has been studied only for the case of bellshaped, noise-free, input wave packets, e.g., for investigating the impact of ST self-phase modulation (SPM) on filament formation, pulse splitting, and related phenomena [8]. The role of the noise has been considered only in the context of $1 \mathrm{D}$ models. The aim of this work is that of providing experimental evidence of the genuine hyperbolic feature of the noise-seeded MI in bulk, normally dispersive, optical systems. To this end we performed experiments and calculations in which, a controlled, broadband noise is injected together with the strong (quasi) plane and monochromatic pump into the system.

The particular system that we have chosen to investigate is the same as in Ref. [14], i.e., that of an optical WP shaped as a large (with respect to diffraction), elongated beam and long (with respect to dispersion) pulse that propagates in a $X^{(2)}$ nonlinear crystal tuned for second harmonic ( $\mathrm{SH}$ ) generation close to phase matching. For the chosen crystal [lithium triborate, (LBO)] and wavelength [first harmonic, 

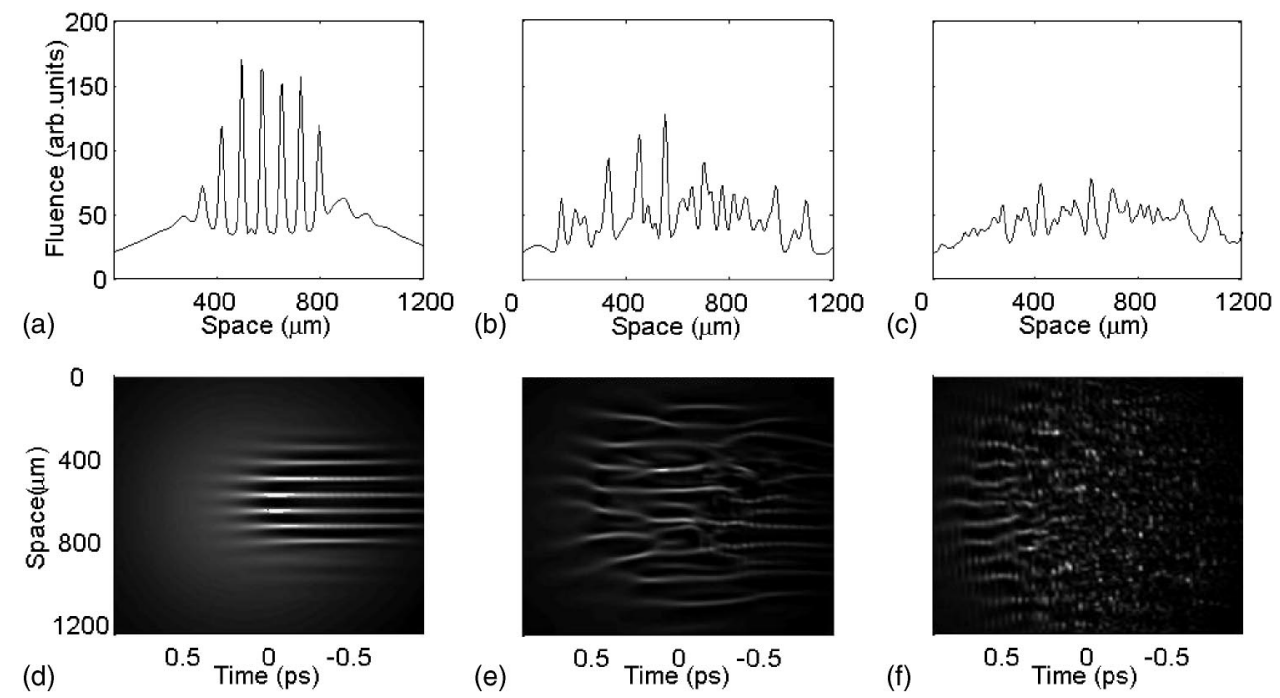

FIG. 1. Calculated space-time profiles (bottom) and corresponding integrated fluence profiles (top) for the fundamental harmonic propagated in a 50-mm lithium triborate (LBO) crystal in regime of $\Delta k=2 k\left(\omega_{0}\right)-k\left(2 \omega_{0}\right)=5 \mathrm{~cm}^{-1}$, in absence of noise (a and d), and with two different kinds of noise: (b and e) $6 \mathrm{~nm}$ and $25 \mathrm{mrad}$, (c and f) $20 \mathrm{~nm}$ and $50 \mathrm{mrad}$ of bandwidth. The intensity level of noise is $1 \%$. The input is a Gaussian FH WP with 1-ps FWHM duration, 600- $\mu$ m FWHM beam width, and 40-GW/ $\mathrm{cm}^{2}$ peak intensity. Calculations are performed in the frame of a $1 \mathrm{D}$ (spatial) $+1 \mathrm{D}$ (temporal) $+1 \mathrm{D}$ (propagation) model. Asymmetry in the temporal coordinate is due to groupvelocity mismatch.

(FH), $1055 \mathrm{~nm}$ ] the chromatic dispersion (see caption to Fig. 1 for details) is such that one should expect MI to take place in the hyperbolic regime [9]. We performed numerical calculations in the frame of a two-dimensional (2D) +1 model integrating [via Fast Fourier transform (FFT), split step, and Runge-Kutta algorithms, with up to 15 -fs $6-\mu \mathrm{m}$ grid and $40-\mu \mathrm{m}$ step] the $\chi^{(2)}$ coupled-wave equations for $\mathrm{FH}$ and $\mathrm{SH}$ envelopes $E_{j}(z, x, t)$,

$$
\begin{gathered}
\hat{L}\left(\omega_{0}\right) E_{1}+\chi E_{2} E_{1}^{*} \exp (-i \Delta k z)=0, \\
\hat{L}\left(2 \omega_{0}\right) E_{2}+i \delta V \partial_{t} E_{2}+\chi E_{1}^{2} \exp (i \Delta k z)=0,
\end{gathered}
$$

where $\hat{L}(\omega) \equiv i \partial_{z}+[2 k(\omega)]^{-1} \partial_{x x}^{2}-\left[k^{\prime \prime}(\omega) / 2\right] \partial_{t t}^{2}, k^{\prime \prime}$ is the group-velocity dispersion (GVD), $\delta V=k^{\prime}\left(2 \omega_{0}\right)-k^{\prime}\left(\omega_{0}\right)$ weighs the group-velocity mismatch (GVM), and $\Delta k$ $=2 k\left(\omega_{0}\right)-k\left(2 \omega_{0}\right)$. Figure 1 gives the calculated fluence (e.g., energy density) profile of the $\mathrm{FH}$ at the output of a $50-\mathrm{mm}$ crystal (top), together with the corresponding ST intensity maps (bottom). Figures 1(a) and 1(d), which refer to noisefree input, show the occurrence of a regular, highly contrasted, spatial breakup of the beam into a spatial-soliton array, which appears as the consequence of MI seeding by the deterministic wave-envelope modulation (WEM [14]).

When the ST noise is injected, WEM and noise-seeded MI compete and the results are those shown in Figs. 1(b), 1(c), 1(e), and 1(f), where the input-noise bandwidth (BW) is increased (from left to right) while keeping fixed the noise intensity at $1 \%$ of the level of the pump. See how the impact of the noise dramatically increases on enlarging its BW, owing to the unbounded instability. Note how the noise, instead of deepening the spatial modulation (as it occurs in the frame of the 1D+1 models [11]), quenches it almost completely. The reason is the appearance of a "chaotic gas" of localized
ST structures [Fig. 1(f)], which gets washed out by the temporal integration. It is worth pointing out that similar results are obtained when keeping the bandwidth fixed and changing the noise intensity.

In order to verify if the outlined catastrophic behavior is a genuine physical effect or an artifact of the approximations adopted, we performed a SH-generation laboratory experiment in similar conditions to those which Fig. 1 refers to. To this end, we used a strongly elongated $(1000 \times 70 \mu \mathrm{m})$, long (1-ps) pump WP, as clean as possible from any spatial or temporal substructure, provided by a chirped pulse amplificated (CPA) Nd:glass laser (TWINKLE, Light Conversion). Then, we superimposed to the pump a weak, broad bandwidth, ST noise, of controllable intensity, generated on a separate channel by a broadband quantum-noise parametric amplifier. For the noise generation we used a 15-mm LBO crystal pumped by the SH of (a portion of) our pump pulse. Both pump and noise WPs were launched synchronously into a 50-mm LBO crystal, tuned for phase-matched SH generation. The spatial and temporal BWs of the noise field at the input of the SH generator were $100 \mathrm{~nm}$ and $60 \mathrm{mrad}$, respectively. Figure 2 (top) shows the fluence distributions of the FH beam at the crystal output facet as recorded by a chargecoupled device (CCD) camera and suitable imaging optics. The corresponding profiles (along the long axis of the beam) are given in Fig. 2 (bottom) for a more quantitative description. The results in the left, center, and right parts of the figure refer to average noise fluence $0,0.01 \%$, and $0.1 \%$ of that of the pump, respectively, for a fixed noise ST BW. The resulting scenario fully confirms the model prediction. Indeed, the noise-induced quenching of the WEM-seeded spatial MI takes place in the experiment for a lower noise level than in calculations, which indicates that the accessible BW is even larger than the computational BW used for obtaining the Figs. 1(c) and 1(f) results. 

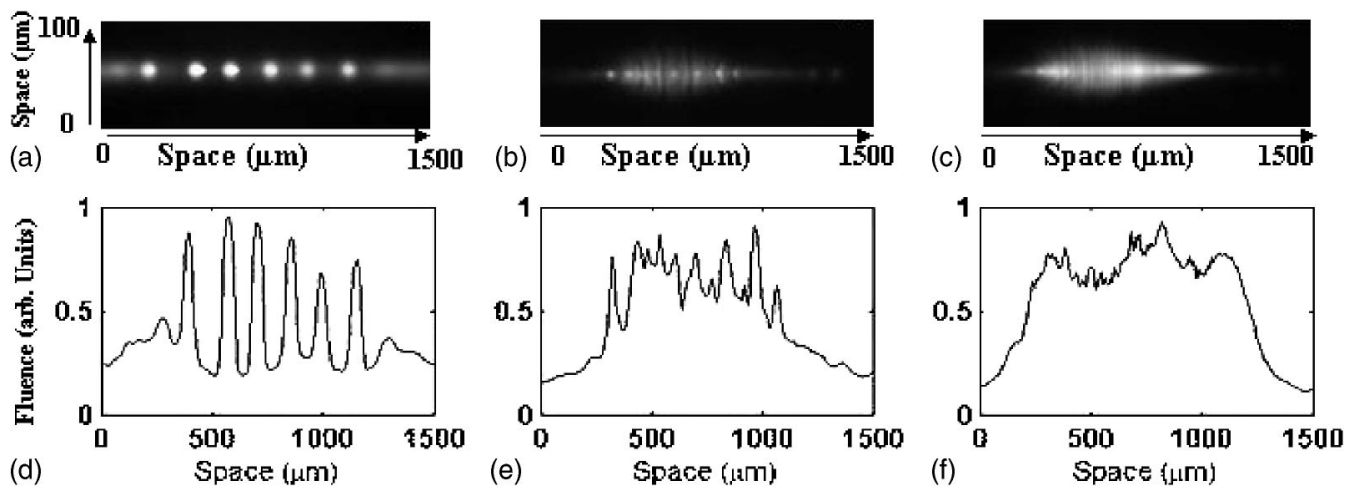

FIG. 2. Measured fluence profiles (top) of FH WP recorded by a CCD camera at the output of the 50-mm LBO crystal, and (bottom) corresponding beam profiles (note that the horizontal scales of images and profiles are not the same) in conditions: (a) and (d) with no noise; (b) and (e) with noise intensity $0.01 \%$ of that of the pump; (c) and (f) with $0.1 \%$ of noise. The noise spatial and temporal BWs were, respectively, of $60 \mathrm{mrad}$ and $100 \mathrm{~nm}$. The input pump intensity was $20 \mathrm{GW} / \mathrm{cm}^{2}$.

The described, near-field, measurements have the obvious limitation of confirming the model prediction only on the basis of a time-integrated effect, the underlying ST structure not being detectable by any technique. Moreover, both the numerical and the experimental results that we have presented do not produce a direct evidence of the hyperbolic nature of instability. In what follows, in order to overcome these limitations, we illustrate the complementary, far-field analysis, concerning the characterization of the angular spec- tra (AS) of the generated field. Figure 3(a) contains the calculated AS (i.e., the square modulus of the field Fourier transform) of a FH profile analogous to that in Fig. 1(f) (see the caption for details). The bright central spot corresponds to the spectrum of the input pulse, while the surrounding structure describes the amplified fluctuations. Note the evident hyperbolic shape of the instability region, which coincides with the region where the calculated MI-gain profile is the largest [9]. We verified that, no matter the size of the
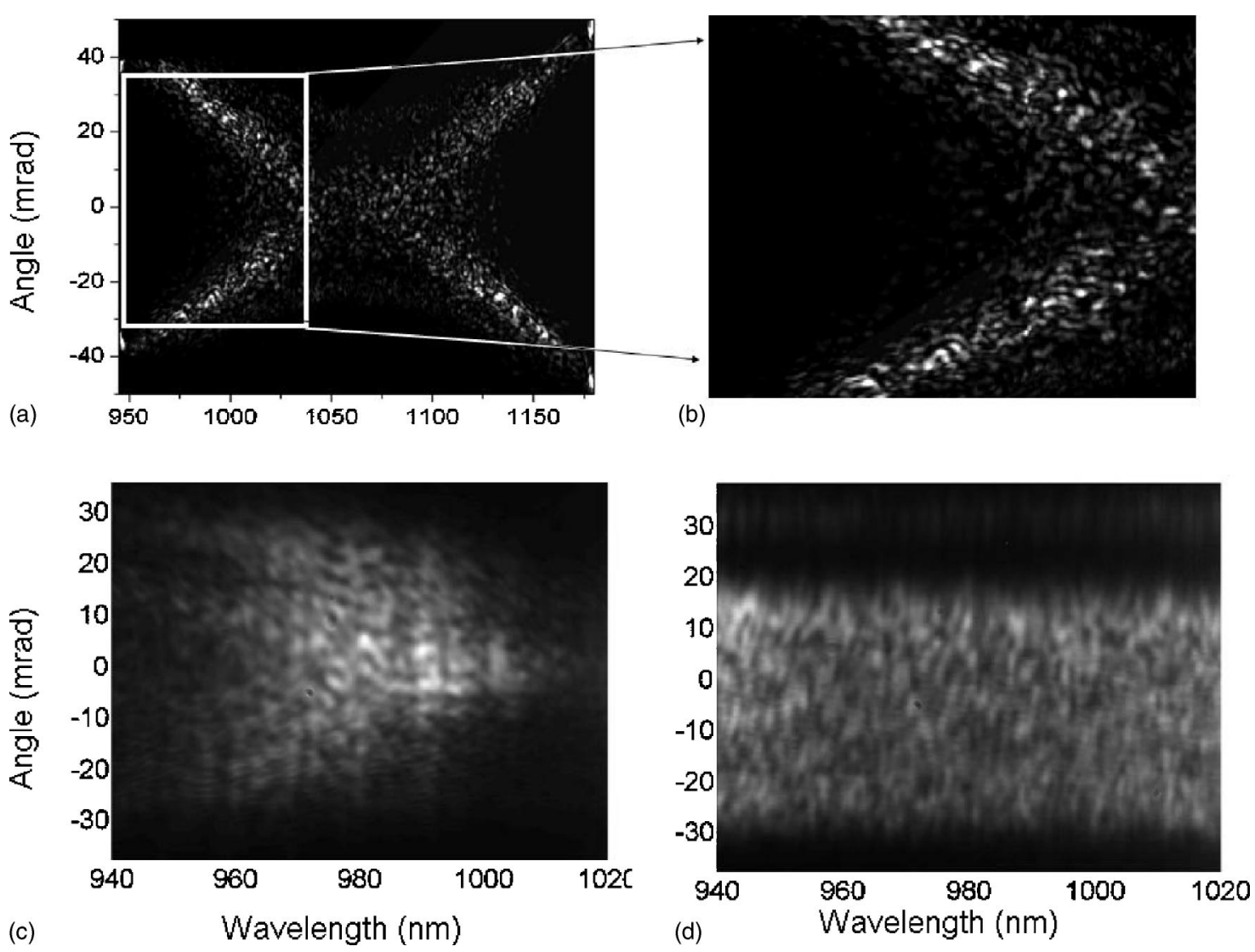

FIG. 3. (a) Calculated angular spectra (AS) of the FH field for operating conditions as those in Fig. 1(f), but for larger input-noise BW (namely: $40 \mathrm{~nm}$ and $50 \mathrm{mrad}$ ); (b) zoom of (a) corresponding to the region of detection; (c) measured AS of the radiation exiting the crystal; (d) measured AS of the input noise [intensity level multiplied by 50 with respect to Fig. 3(c)]. The gray colors refer to logarithmic scale. 
Fourier space, the instability always reaches the border of the spectral box (in case of large enough noise BW), thus confirming the unbounded nature of the MI process in the frame of the adopted model. For the far-field experiment, we measured the FH AS by placing the entrance slit of a largenumerical-aperture imaging spectrometer at the focal plane of a positive lens. The detected AS in the short-wavelength branch of the spectrum (the long-wavelength one is not accessible due to sensitivity cut off of the silicon CCD detector) is reported in Fig. 3(c). The detected portion of the AS (the radiation at large angles was clipped by the very narrow aperture of our $3 \times 3 \times 50 \mathrm{~mm}^{3}$ LBO crystal) exhibits a good qualitative agreement with calculations [see the zoom in Fig. $3(b)]$ and confirms the genuine hyperbolic feature of the instability process.

In conclusion, in the regime of second-harmonic generation with normal dispersion, by superimposing on an intense, clean, pump wave packet and a weak spatiotemporal noise (with coherence time much shorter than that of the pump) we have shown that the noise-seeded MI develops in the spatiotemporal domain. Due to the unbounded feature of the instability, and so the large response of the system to white noise, a noise as weak as $0.01 \%$ of the pump leads to quenching of the spatial deterministic beam breakup and spatial-soliton formation caused by wave-envelope modulation. The analysis of the results in the spectral domain outlined the genuine hyperbolic feature of the instability, which couples different frequencies to different angles. Because of this coupling, the quenching of detectable spatial effects cannot be simply interpreted as the averaging of several inde- pendent spatial structures, occurring for different time slices of the wave packet. As pointed out in [12], the typical (amplitude and phase) fluctuations that were triggering the instability in previous experiments (without external noise injection) were probably caused by laser beam, or opticalcomponent or nonlinear sample imperfections, thus leading to a "frozen noise" with the same coherence time of the pump (that virtually coincides with the pulse duration, all lasers operating close to the transform limit). This might explain why the resulting instability was described in the frame of monochromatic models. Finally, we expect that the described, hyperbolic instability should play a dramatic role when the field is strong enough to probe vacuum state fluctuations, which indeed provide the source of a virtually $\delta$-correlated noise. We expect that quantum-noise seeded hyperbolic instability should dominate not only the $X^{(2)}$ parametric-amplification regime (as evident, for example, in Ref. [15] and also in the more recent Ref. [16], but also the classical, unseeded, second-harmonic generation process and, possibly, the Kerr regime too. This might explain the "spontaneous" quenching of spatial breakup seen at high pumping in $[12,14]$. Owing to the unbounded nature of the instability, the robustness of nonlinear dynamics of normally dispersive media with respect to the interaction with the quantum noise represents, therefore, a crucial issue which deserves further investigation.

The authors acknowledge support from Contracts No. FIRB 2001 (Italy) and No. DGICYT BFM2002-04369C04-03 (Spain).
[1] B. T. Benjamin and J. E. Feir, J. Fluid Mech. 27, 417 (1967).

[2] T. Kakutami and N. Sugimoto, Astrophys. Space Sci., 159, 347 (1974).

[3] J. M. Bilbault, P. Marquie, and B. Michaux, Phys. Rev. E 51, 817 (1995).

[4] U. Al Khawaja, H. T. C. Stoof, R. G. Hulet, K. E. Strecker, and G. B. Partridge, Phys. Rev. Lett. 89, 200404 (2002).

[5] V. I. Bespalov and V. I. Talanov, JETP Lett. 3, 307 (1966).

[6] D. Jaksch, C. Bruder, J. I. Cirac, C. W. Gardiner, and P. Zoller, Phys. Rev. Lett. 81, 3108 (1998).

[7] L. W. Liou, X. D. Cao, C. J. McKinstrie, and G. P. Agrawal, Phys. Rev. A 46, 4202 (1992).

[8] G. G. Luther, A. C. Newell, J. V. Moloney, and E. M. Wright, Opt. Lett. 19, 789 (1994).

[9] S. Trillo, C. Conti, P. Di Trapani. O. Jedrkiewicz, J. Trull, G. Valiulis, and G. Bellanca, Opt. Lett. 27, 1451 (2002).
[10] K. Tai, A. Hasegawa, and A. Tomita, Phys. Rev. Lett. 56, 135 (1986).

[11] R. A. Fuerst, D.-M. Baboiu, B. Lawrence, W. E. Torruellas, G. I. Stegeman, S. Trillo, and S. Wabnitz, Phys. Rev. Lett. 78, 2756 (1997).

[12] H. Fang, R. Malendevich, R. Schiek, and G. Stegeman, Opt. Lett. 25, 1786 (2001).

[13] R. Malendevich, L. Jankovic, G. Stegeman, and J. S. Aitchison, Opt. Lett. 26, 1879 (2001).

[14] D. Salerno, S. Minardi, J. Trull, A. Varanavicius, G. Tamosauskas, G. Valiulis, A. Dubietis, D. Caironi, S. Trillo, A. Piskarskas, and P. Di Trapani, Phys. Rev. Lett. 91, 143905 (2003).

[15] F. Devaux and E. Lantz, J. Opt. Soc. Am. B 12, 2245 (1995).

[16] H. Zeng, J. Wu, H. Xu, K. Wu, and E. Wu, Phys. Rev. Lett. 92, 143903 (2004). 